\begin{tabular}{|c|l|}
\hline Title & Temperature dependence of band gap energies of GaA sN alloys \\
\hline Author(s) & Uesugi, Katsuhiro; Suemune, Ikuo; Hasegawa, Tatsuo; A kutagawa, Tomoyuki; Nakamura, Takay oshi \\
\hline Citation & $\begin{array}{l}\text { A pplied Physics Letters, 76(10), 1285-1287 } \\
\text { https://doi.org/10.1063/126010 }\end{array}$ \\
\hline Issue Date & 2000-03-06 \\
\hline Doc URL & http://hdl.handle.net/2115/5574 \\
\hline Rights & Copyright $\odot 2000$ A merican Institute of Physics \\
\hline Type & article \\
\hline File Information & APL 76-10.pdf \\
\hline
\end{tabular}

Instructions for use 


\title{
Temperature dependence of band gap energies of GaAsN alloys
}

\author{
Katsuhiro Uesugi, Ikuo Suemune, ${ }^{\text {a) }}$ Tatsuo Hasegawa, Tomoyuki Akutagawa, \\ and Takayoshi Nakamura \\ Research Institute for Electronic Science, Hokkaido University, Sapporo 060-0812, Japan
}

(Received 17 August 1999; accepted for publication 14 January 2000)

\begin{abstract}
The temperature dependence of band gap energies of GaAsN alloys was studied with absorption measurements. As the $\mathrm{N}$ concentration in GaAsN increased, the temperature dependence of the band gap energy was clearly reduced in comparison with that of GaAs. The redshift of the absorption edge in GaAsN for the temperature increase from 25 to $297 \mathrm{~K}$ was reduced to $60 \%$ of that of GaAs for the $\mathrm{N}$ concentration larger than $\sim 1 \%$. The differential temperature coefficient of the energy gap at room temperature was also reduced to $70 \%$ of that of GaAs. The main factor for this reduced temperature dependence in GaAsN was attributed to the transition from band-like states to nitrogen-related localized states with detailed studies of the temperature-induced shift of the absorption edge. (C) 2000 American Institute of Physics. [S0003-6951(00)04110-3]
\end{abstract}

III-V-N alloys such as GaAsN and GaInNAs have been intensively studied to realize long-wavelength semiconductor lasers with higher temperature stability for optical-fiber communications. ${ }^{1}$ Although $1.3 \mu \mathrm{m}$ semiconductor lasers based on the InGaAsP/InP system have been commonly used, their lasing threshold currents are highly temperature dependent due to poor carrier confinements in InGaAsP quantum wells. GaInNAs/AlGaAs system grown on GaAs substrates will be able to confine electrons more tightly in the quantum wells due to the larger conduction bands offsets compared with the InGaAsP system, which will efficiently prevent leakage currents and will reduce the temperature dependence of the threshold currents. ${ }^{1}$ The higher temperature stability of the lasing threshold was demonstrated in a GaInNAs laser operating at around $1.3 \mu \mathrm{m} .^{2}$

In addition to the stability of the lasing threshold against the environment change, the stability of the lasing wavelength is another important requirement on laser light sources for optical-fiber communications. Perlin et al. ${ }^{3}$ recently studied the pressure and temperature dependence of the absorption edge of a $\mathrm{Ga}_{0.92} \mathrm{In}_{0.08} \mathrm{~N}_{0.015} \mathrm{As}_{0.985}$ alloy. They reported that the hydrostatic pressure coefficient of the band gap of the GaInNAs was more than a factor of 2 lower than that of GaAs. The temperature-induced shift of the absorption edge of GaInNAs was $12 \%$ smaller than that of GaAs. They explained this phenomenon by the large decrease of the deformation potential.

In this letter, the relation between the temperature dependence of the band gap energy of the GaAsN alloys and their $\mathrm{N}$ concentration is studied. A substantial decrease of the temperature dependence was found in GaAsN, and the transition from the temperature dependence of GaAs band-like states to that of more localized states was observed for the higher $\mathrm{N}$ concentrations.

$0.1-6.2-\mu \mathrm{m}$-thick GaAsN films were grown on semiinsulating $\mathrm{GaAs}(001)$ substrates by metalorganic molecularbeam epitaxy. The metalorganic precursors used were triethylgallium, monomethylhydrazine, and trisdimethylami-

${ }^{a)}$ Electronic mail: isuemune@es.hokudai.ac.jp noarsenic (TDMAAs). After thermal cleaning of GaAs substrate surfaces at $600{ }^{\circ} \mathrm{C}$ with the simultaneous supply of TDMAAs, GaAsN layers were grown at $520-570{ }^{\circ} \mathrm{C}$. The $\mathrm{N}$ concentration in the GaAsN films were estimated from highresolution x-ray diffraction measurements. Detailed growth conditions and the estimation of the solid concentrations in GaAsN are reported elsewhere. ${ }^{4,5}$ The optical properties of the GaAsN films were studied by absorption measurements in the temperature range of 25-297 K.

Figure 1 shows the photon energy dependence of $(\alpha E)^{2}$ of GaAs, $\mathrm{GaAs}_{0.9966} \mathrm{~N}_{0.0034}$, and $\mathrm{GaAs}_{0.992} \mathrm{~N}_{0.008}$ evaluated from the transmission spectrum measurements at 25 and 297 $\mathrm{K}$, where $\alpha$ is the absorption coefficient in $\mathrm{cm}^{-1}$ and $E$ is the photon energy in $\mathrm{eV}$. The band gap energies of GaAs and GaAsN alloys were estimated by extrapolating the linear part of $(\alpha E)^{2}$ to zero. With the increase of the $\mathrm{N}$ concentration in GaAsN, the measured band gap energies were shifted to the lower energy. The band gap energies are redshifted with the

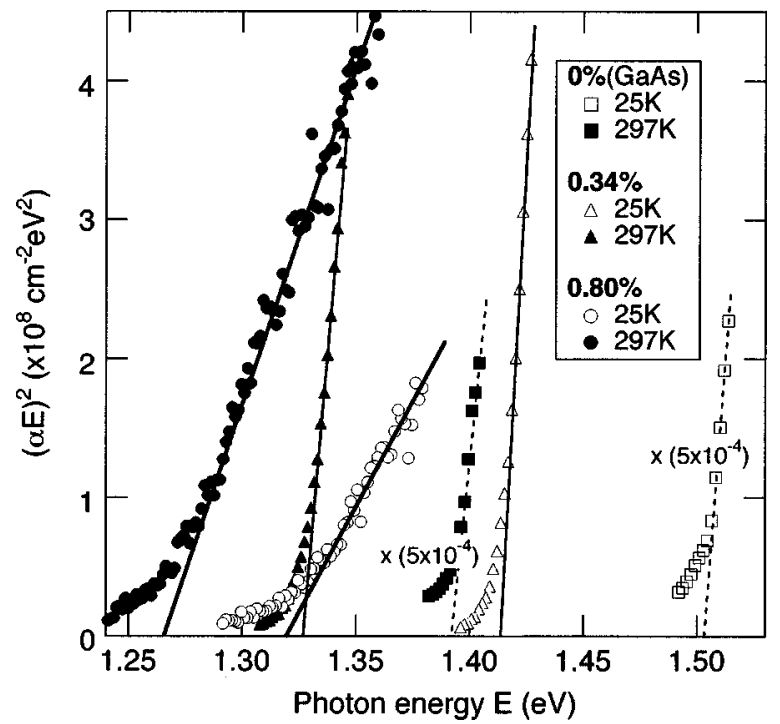

FIG. 1. Photon energy dependence of $(\alpha E)^{2}$ of GaAsN with various $\mathrm{N}$ concentrations $(0 \%, 0.34 \%$, and $0.80 \%)$ measured at 25 and $297 \mathrm{~K}$. The band gap energies of the films were estimated by extrapolating the linear part of $(\alpha E)^{2}$ to zero. 


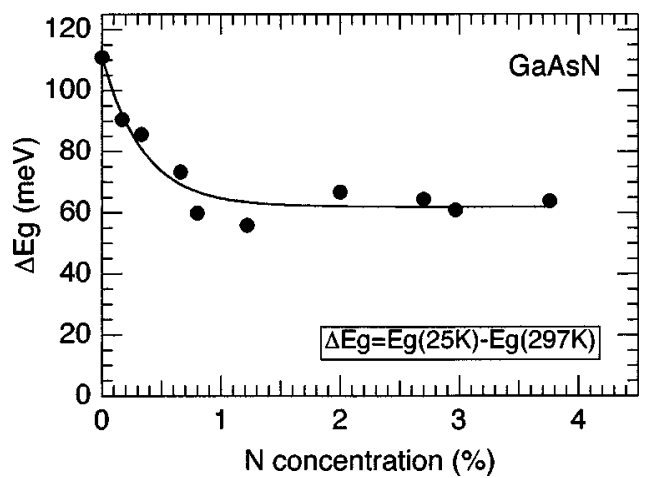

FIG. 2. N concentration dependence of the band gap shift of the GaAsN alloys when the temperature is changed from 25 to $297 \mathrm{~K}$.

temperature rise from 25 to $297 \mathrm{~K}$, but it will be clear that the redshift energy difference is reduced for the higher $\mathrm{N}$ concentration.

Figure 2 shows the $\mathrm{N}$ concentration dependence of the band gap shift $\left(\Delta E_{g}\right)$ with the temperature change between 25 and $297 \mathrm{~K}$. As the $\mathrm{N}$ concentration in GaAsN is increased, $\Delta E_{g}$ is rapidly decreased. Above the $\mathrm{N}$ concentration of $0.80 \%, \Delta E_{g}$ is nearly concentration independent and is about $60 \%$ of that of GaAs. This result suggests that the temperature-induced band gap shift is changing from that of GaAs to the one of N-related states.

To study the origin of this $\mathrm{N}$ concentration dependence, the temperature dependence of the energy gap was measured in more detail for various $\mathrm{N}$ concentrations up to $\sim 4 \%$. Figure 3 summarizes the temperature dependence of the measured band gap energies of the GaAsN films. It is well known that the temperature dependence of the direct band gap energy can be described by the Bose-Einstein expression proposed by Lautenschlager et al. ${ }^{6}$ or the Varshni equation. ${ }^{7}$ The dashed line (a) and the solid line (b) are the fits to the measured data of GaAs and GaAsN with the $2.97 \%$ concentration with the Bose-Einstein expression, respectively. The Varshni equation also gives good fits to the measurements and the parameters used for the fitting are summarized in Table I. The differential temperature coefficient $d E_{g} / d T$ measured at room temperature is also shown in Table I.

The dashed line (a) and the solid line (b) were vertically shifted by constant energies to check whether these lines can also fit the other $\mathrm{N}$ concentrations. When the $\mathrm{N}$ concentration is in the range of $0.80 \%-3.76 \%$, the measured temperature dependence was nicely fitted with the vertically shifted solid line (b) as shown in Fig. 3. This is consistent with the tendency shown in Fig. 2. However, the properties for the $\mathrm{N}$ concentration range of $0.17 \%-0.66 \%$ could not be fitted with the single fitting model. The temperature dependence in the

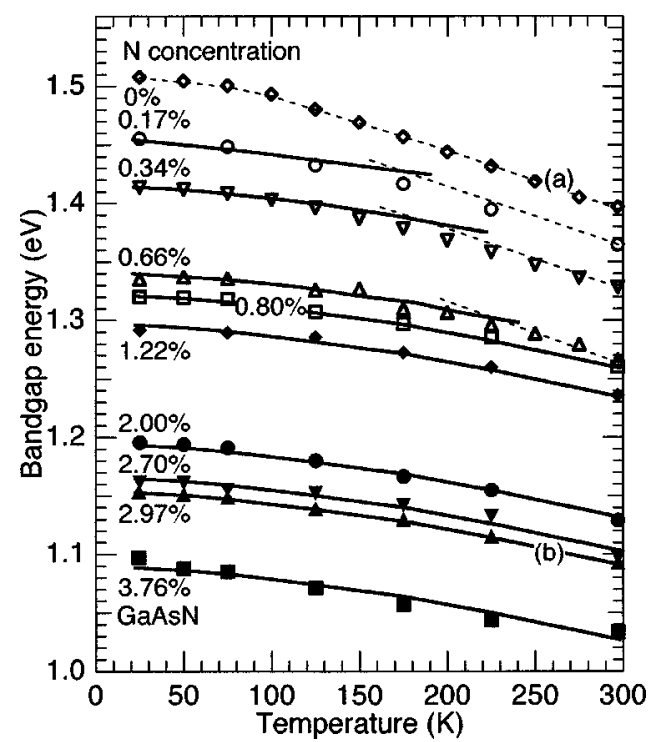

FIG. 3. Temperature dependence of the measured band gap energies of the GaAsN films. The $\mathrm{N}$ concentrations in GaAsN are $0 \%-3.76 \%$.

lower temperature range could be fitted with the vertically shifted solid line (b), while that in the higher temperature range near room temperature was more close to the vertically-shifted dashed line (a). The specific temperature where the fittings with the solid and dashed lines cross was shifted to the higher energy for the higher $\mathrm{N}$ concentration.

These results show that two kinds of N-related energy states with the different temperature dependencies play roles in determining the temperature dependence of the GaAsN band gap energies, i.e., an energy state with the higher temperature dependence similar to the GaAs bands and a $\mathrm{N}$-related localized state with the lower temperature dependence. The former band-like state will be located in the higher energy at low temperature. But this state will show the larger redshift with the temperature rise and will cross over the localized impurity-like state which is located in the lower energy at the lower temperature.

Recently, the redshift of band gap energies in GaInNAs alloys induced by the $\mathrm{N}$ inclusion was discussed with a band anticrossing model assuming the formation of a narrow resonant band formed by nitrogen states which interact with the conduction band. ${ }^{8}$ The pressure dependence of the band gap energies measured on the GaInNAs alloys showed the transition from the linear dependence to the much weaker dependence for the higher pressure. This pressure dependence was explained by considering two interacting energy levels: one associated with extended states and the other with the localized $\mathrm{N}$ states. The two energy states with the different temperature dependence discussed previously is consistent with

TABLE I. Values of the parameters $E_{0}, \alpha$, and $\beta$ obtained by fitting to the Varshni relation (Ref. 7) $E_{g}(T)$ $=E_{0}-\alpha T^{2} /(\beta+T)$ and $E_{B}, \alpha_{B}$, and $\Theta_{B}$ obtained by fitting to the Bose-Einstein expression (Ref. 6) $E_{g}(T)=E_{B}-\alpha_{B}\left\{1+2 /\left[\exp \left(\Theta_{B} / T\right)-1\right]\right\}$.

\begin{tabular}{ccccccccc}
\hline \hline Sample & $\begin{array}{c}\mathrm{N} \text { concentration } \\
(\%)\end{array}$ & $\begin{array}{c}E_{0} \\
(\mathrm{eV})\end{array}$ & $\begin{array}{c}\alpha \\
(\mathrm{meV} / \mathrm{K})\end{array}$ & $\begin{array}{c}\beta \\
(\mathrm{K})\end{array}$ & $\begin{array}{c}E_{B} \\
(\mathrm{eV})\end{array}$ & $\begin{array}{c}\alpha_{B} \\
(\mathrm{meV})\end{array}$ & $\begin{array}{c}\Theta_{B} \\
(\mathrm{~K})\end{array}$ & $\begin{array}{c}d E_{g} / d T \times 10^{4} \\
(\mathrm{eV} / \mathrm{K})^{\mathrm{a}}\end{array}$ \\
\hline (a) & 0 & 1.512 & 0.56 & 146 & 1.545 & 37 & 152 & -5.0 \\
$(\mathrm{~b})$ & 2.97 & 1.154 & 0.61 & 560 & 1.202 & 49 & 285 & -3.1 \\
\hline \hline
\end{tabular}

${ }^{2} 200-300 \mathrm{~K}$. 
these two energy levels assumed in the band anticrossing model. The fundamental key factor for this localized state formation is the large differences between atomic orbital energies and sizes of the alloyed As and $\mathrm{N}$ atoms. ${ }^{9}$

The temperature, induced shift of the absorption edge in GaAsN was reduced by $40 \%$ compared with that of GaAs in this work, while it was reduced by only $12 \%$ in GaInNAs with the $\mathrm{N}$ concentration of $1.5 \%{ }^{3}$ This may be due to the lowering of the band-like GaInAs state energy by the inclusion of In. This will shift the cross-over temperature discussed in Fig. 3 to the lower temperature, and the GaInAs band-like state will be more dominant up to the higher $\mathrm{N}$ concentration, resulting in the smaller effect of $\mathrm{N}$ on the temperature induced shift of the band gap energies.

In conclusion, the temperature dependence of the band gap energy of GaAsN films were studied by absorption measurements. The temperature induced shift of the band gap energy in GaAsN was substantially reduced by the presence of $\mathrm{N}$ in GaAs. This reduced temperature dependence of the band gap energy was discussed considering the N-related localized energy states with the lower temperature depen- dence and the band-like energy states with the temperature dependence close to that of GaAs.

The authors are grateful to Mitsuo Hoshiyama for his technical support in this work. This work was supported in part by Telecommunications Advancement Organization of Japan.

\footnotetext{
${ }^{1}$ M. Kondow, K. Uomi, A. Niwa, T. Kitatani, S. Watahiki, and Y. Yazawa, Jpn. J. Appl. Phys., Part 1 35, 1273 (1996).

${ }^{2}$ K. Nakahara, M. Kondow, T. Kitatani, M. C. Larson, and K. Uomi, IEEE Photonics Technol. Lett. 10, 487 (1998).

${ }^{3}$ P. Perlin, S. G. Subramanya, D. E. Mars, J. Kruger, N. A. Shapiro, H. Siegle, and E. R. Weber, Appl. Phys. Lett. 73, 3703 (1998).

${ }^{4}$ K. Uesugi and I. Suemune, Jpn. J. Appl. Phys., Part 2 36, L1572 (1997).

${ }^{5}$ K. Uesugi, N. Morooka, and I. Suemune, Appl. Phys. Lett. 74, 1254 (1999).

${ }^{6}$ P. Lautenschlager, M. Garriga, S. Logothetidis, and M. Cardona, Phys. Rev. B 35, 9174 (1987).

${ }^{7}$ Y. P. Varshni, Physica (Utrecht) 34, 149 (1967).

${ }^{8}$ W. Shan, W. Walukiewicz, J. W. Ager III, E. E. Haller, J. F. Geisz, D. J. Friedman, J. M. Olson, and S. P. Kurtz, Phys. Rev. Lett. 82, 1221 (1999).

${ }^{9}$ S. H. Wei and A. Zunger, Phys. Rev. Lett. 76, 664 (1996).
} 